\title{
Air-to-Ground Attack Decision-Making of Controlling Trajectory Based on RHC-PSO
}

\author{
WANG Zhi-hong ${ }^{a, *}$, WEI Xian-zhi and ZHENG Xiao-fei ${ }^{\mathrm{b}}$ \\ ${ }^{1}$ Engineering College of Aeronautics and Astronautics, Air Force Engineering University, \\ Xi'an, China \\ a wangzhihong7285829@163.com, ${ }^{\mathrm{b}}$ zxf2655640@163.com \\ *corresponding author
}

Keywords: air-to-ground attack, control of trajectory, RHC-PSO, aid decision making

\begin{abstract}
Aiming at the controlling of trajectory during the air-to-ground attack, firstly, the 6-DOF model of combat aircraft and the model of the threats are established. The thrust coefficient, angleof-attack, and roll angle are chosen as the controlled quantity. Then establish the indicator function and the optimum control modelling of the air-to-ground trajectory decision-making. Adopt the RHC-PSO algorithm, getting the controlling quantity's optimum value in every decision-making time domain, combining the RHC to realize online controlling of trajectory. Simulation shows that the algorithm can control the aircraft to perform the operational mission and show the change law of the controlled quantity, giving the information of aid decision making to pilot.
\end{abstract}

\section{Introduction}

In the air-ground fighting process, the environment of battlefield is extraordinarily complicated, which brings huge pressure to pilot. Thus, when controlling the trajectory of fighter, it is not enough to only plan one suitable airway. With the progress of fighting, the change law of the controlled quantity of fighter manipulated by pilot is also needed to be planned so as to provide aid decision making information for the attack trajectory control of fighter, reduce the manipulation pressure of pilot and enhance the operational effectiveness.

For trajectory control decision problem, the currently adopted methods includes artificial intelligence algorithm, such as $\mathrm{A}^{*}$ algorithm, genetic algorithm (GA), particle swarm optimization (PSO), etc., which was mainly used for the track planning. However, traditional intelligence algorithm is difficult to implement real-time control of airway as well as the change law of controlled quantity. Gauss pseudo-spectral method (GPM) ${ }^{[1]}$, receding pseudo-spectral method $(\mathrm{RPM})^{[2]}$ transform the trajectory control to the optimal control problem of a continuous system and transform it further to discrete parameter optimization problem by adopting pseudo-spectral method (PM) so as to solve the optimal controlled quantity at each discrete point. Then, the optimal controlled quantity of more discrete point is got through interpolation. When it is necessary to enhance the trajectory control precision, the quantity of discrete point must be increased so as to affect the initial setting and algorithm performance.

Based on this, the paper firstly processes the trajectory planning using discretion method, then introduces receding horizon control (RHC), utilizes particle swarm optimization (PSO) to optimize the controlled quantity of fighter at discrete moment, acts the acquired optimal control sequence on the system and updates the horizon receding so as to solve the optimal controlled quantity sequence at each decision moment, which solves the air-ground trajectory control decision problem. 


\section{Establishment of Basic Model}

\subsection{Six Degrees of Freedom Model of Fighter}

The mass of fighter is supposed to be a constant, the installation angle of motor is $0^{\circ}$, the angle of attack and sideslip is 0 , and then the kinematical equation of fighter is:

$$
\left\{\begin{array}{l}
\dot{x}_{f}=v_{f} \cos \theta_{f} \cos \varphi_{f} \\
\dot{y}_{f}=v_{f} \cos \theta_{f} \sin \varphi_{f} \\
\dot{z}_{f}=v_{f} \sin \theta_{f}
\end{array}\right.
$$

In the equation, $v_{f} 、 \theta_{f}, \varphi_{f}$ is the velocity of fighter, angle of pitch and deflection angle of trajectory.

Kinetic equation of fighter is:

$$
\left\{\begin{array}{l}
\dot{\theta}_{f}=\frac{1}{m_{f} v_{f}}\left\{\left(L+\eta T_{\max } \sin \alpha_{f}\right) \cos \mu_{f}-m_{f} g \cos \theta_{f}\right\} \\
\dot{\varphi}_{f}=\frac{1}{m_{f} v_{f} \cos \theta_{f}}\left(L+\eta T_{\max } \sin \alpha_{f}\right) \sin \mu_{f} \\
\dot{v}_{f}=\frac{1}{m_{f}}\left(\eta T_{\max } \cos \alpha_{f}-D_{z}\right)-g \sin \theta_{f}
\end{array}\right.
$$

In the equation, $m_{f}$ is the mass of fighter, $g$ is the gravitational acceleration, and $\eta$ is thrust coefficient; $T_{\max }$ is the maximal available thrust; $\alpha_{f}$ is angle of attack (AOA); $\mu_{f}$ is roll angle; lift force is $L=q S C_{L}$; resistance $D_{Z}=q S C_{D}$; kinetic pressure $q=\rho v^{2} / 2, \rho$ is air density; $S$ is wing area. For the computing method of the above variables, please refer to Literature ${ }^{[3]}$.

To enhance the computation speed, variable and quantity of state is procseed using dimensionless method ${ }^{[4]}$ :

$$
\left\{\begin{array}{l}
\bar{v}=\frac{v}{v_{s}} \\
\bar{x}=\frac{g x}{v_{s}^{2}}, \bar{y}=\frac{g y}{v_{s}^{2}}, \bar{z}=\frac{g z}{v_{s}^{2}} \\
\bar{t}=\frac{t}{t_{c}} \\
\bar{\gamma}=\frac{\gamma}{\pi}, \bar{\chi}=\frac{\chi}{\pi}, \bar{\alpha}=\frac{\alpha}{\pi / 2}, \bar{\mu}=\frac{\mu}{\pi / 2}
\end{array}\right.
$$

Hereinto, $v_{s}$ expresses velocity of sound; $t_{c}$ expresses time constant, the size is determined according to the situation. Thus, we get: 


$$
\left\{\begin{array}{l}
\frac{d \bar{x}}{d \bar{t}}=\frac{g t_{c} \bar{v}}{v_{s}} \cos (\bar{\gamma} \pi) \cos (\bar{\chi} \pi) \\
\frac{d \bar{y}}{d \bar{t}}=\frac{g t_{c} \bar{v}}{v_{s}} \cos (\bar{\gamma} \pi) \sin (\bar{\chi} \pi) \\
\frac{d \bar{z}}{d \bar{t}}=\frac{g t_{c} \bar{v}}{v_{s}} \sin (\bar{\gamma} \pi) \\
\frac{d \bar{v}}{d \bar{t}}=\frac{t_{c}}{v_{s}} \cdot \frac{\eta T_{\max } \cos \left(\bar{\alpha} \frac{\pi}{2}\right)-D-m g \sin (\bar{\gamma} \pi)}{m} \\
\frac{d \bar{\gamma}}{d \bar{t}}=\frac{t_{c}}{\pi v_{s}} \cdot \frac{\left(L+T \sin \left(\bar{\alpha} \frac{\pi}{2}\right)\right) \cos \left(\bar{\mu} \frac{\pi}{2}\right)-m g \cos (\bar{\gamma} \pi)}{m \bar{v}} \\
\frac{d \bar{\chi}}{d \bar{t}}=\frac{t_{c}}{\pi v_{s}} \cdot \frac{\left(L+\eta T_{\max } \sin \left(\bar{\alpha} \frac{\pi}{2}\right)\right) \sin \left(\bar{\mu} \frac{\pi}{2}\right)}{m \bar{v} \cos (\bar{\gamma} \pi)}
\end{array}\right.
$$

The value range of variables is as below:

$$
\left\{\begin{array}{l}
0 \leq \eta \leq 1 \\
\alpha_{\min } \leq \alpha \leq \alpha_{\max } \\
\mu_{\text {min }} \leq \mu \leq \mu_{\max } \\
v_{\text {min }} \leq v \leq v_{\max } \\
\gamma_{\text {min }} \leq \gamma \leq \gamma_{\max } \\
\chi_{\text {min }} \leq \chi \leq \chi_{\max }
\end{array}\right.
$$

\subsection{Threat Constraint Model}

When executing air-ground defense penetration task, to avoid touching the ground, the flight height should meet following formula:

$$
z-h(x, y) \geq h_{\min }
$$

Hereinto, $h(x, y)$ expresses the terrain height; $h_{\min }$ expresses minimal liftoff safety height.

About ground radar detection threat, the posture of aerial carrier is different for the radar, the RCS is different, and the distance detected by radar is also different, which can be expressed as:

$$
\left\{\begin{array}{l}
R_{i}=f\left(\sigma_{i}\right) \\
\sigma_{i}=g(\alpha, \mu, \gamma, \chi)
\end{array}\right.
$$

Hereinto, $R_{i}$ expresses the operating distance of $i$ th threat; $\sigma_{i}$ expresses RCS value of aerial carrier for threat $i$.

\subsection{Weapon launching constraint model}

The purpose of trajectory control is to manipulate the flight posture of aerial carrier through controlling various controlled quantities so that the aerial carrier arrives at the drop zone of weapon at the maximum survival probability and complete effective attack of goal. Thus, the posture of aerial carrier should meet the drop condition of corresponding weapon. The constraint model is as below:

$$
\left\{\begin{array}{l}
\left(x_{f}, y_{f}, z_{f}\right) \in \delta_{F} \\
\alpha_{f} \in\left[\alpha_{F \min }, \alpha_{F \max }\right], \mu_{f} \in\left[\mu_{F \min }, \mu_{F \max }\right] \\
v_{f} \in\left[v_{F \min }, v_{F \max }\right]
\end{array}\right.
$$


Hereinto, $\left(x_{f}, y_{f}, z_{f}\right)$ expresses the coordinate of fighter at the end point of planning; $\delta_{F}$ expresses the available launching zone of the selected weapon; $\left[\alpha_{F \min }, \alpha_{F \max }\right],\left[\mu_{F \min }, \mu_{F \max }\right]$ and $\left[v_{F \min }, v_{F \max }\right]$ express the end point of planning, AOA of aerial carrier, roll angle and allowable range of velocity.

\section{Trajectory Control Decision Model}

\subsection{Trajectory Control Decision Model}

Transformation of trajectory control decision problem to an optimal control problem needed to establish indicator function reflecting fighting task. Combined with the fighting practice, it is expressed as below ${ }^{[5]}$ :

$$
\begin{gathered}
\min J=t_{c} \int_{\bar{t}_{0}}^{\bar{t}_{f}} g(\mathbf{x}, \mathbf{u}, t) d \bar{t} \\
g(\mathbf{x}, \mathbf{u}, t)=-W_{1} \bar{v}(\bar{t})+W_{2} \bar{\gamma}^{2}+W_{3} \bar{\chi}^{2}+W_{4}\left(\bar{z}-g h(x, y) / v_{s}^{2}\right)^{2}
\end{gathered}
$$

Hereinto, $\mathrm{x}$ and $\mathrm{u}$ express the quantity of state and controlled quantity of aerial carrier, and we easily get $\mathbf{x}=(x, y, z, v, \gamma, \chi)^{T}$ and $\mathbf{u}=(\alpha, \mu, \eta)^{T} ; \bar{t}_{0}=t_{0} / t_{c}, \bar{t}_{f}=t_{f} / t_{c} . t_{0}$ and $t_{f}$ express the moment at the starting and end of planning; the first item on the right side of Equation (10) expresses the air range as small as possible, the second item and third item express the trajectory as smooth as possible, and the fourth item express flight height as low as possible. $W_{1}, W_{2}, W_{3}$ and $W_{4}$ express the weight of each item.

\subsection{Optimal Control Model of Air-Ground Attach Trajectory Plan Decision}

Through indicator function established in 3.1 and flight state model and various constraint model in Section 2, we can get the following optimal control model of air-ground trajectory control decision as:

$$
\begin{array}{ll}
\min & J(\mathbf{x}, \mathbf{u})=t_{c} \int_{\bar{t}_{0}}^{\bar{t}_{f}} g(\mathbf{x}(\bar{t}), \mathbf{u}(\bar{t}), \bar{t}) d \bar{t} \\
s t \quad & \dot{\mathbf{x}}(\bar{t})=f[\mathbf{x}(\bar{t}), \mathbf{u}(\bar{t}), \bar{t}] \\
& \mathbf{x}\left(\bar{t}_{0}\right)=\mathbf{x}_{\mathbf{0}}, \quad \mathbf{x}\left(\bar{t}_{f}\right)=\mathbf{x}_{f} \\
& s_{j}(\mathbf{x}(t), \mathbf{u}(t), t) \leq 0,(j=1,2, \cdots, l) \\
& \bar{t} \in\left[\bar{t}_{0}, \bar{t}_{f}\right]
\end{array}
$$

Hereinto, target function corresponds to Equation (9); state equation corresponds to Equation (4); boundary value contained initial condition and end point constraint; and inequation corresponds to constraint condition, including threat constraint and value range of various variables.

\section{Air-to-Ground Attack Decision-Making of Controlling Trajectory Based on RHC-PSO}

\subsection{Algorithm Description}

The basic thought of RHC is: make discrete sampling of the planned horizon $\left[t_{k}, t_{k}+T\right]$, select discrete point $\left[t_{k}^{1}, t_{k}^{2}, \cdots, t_{k}^{N}\right]$. When online control is carried, we take the state $x\left(t_{k}^{i}\right)$ of system at each discrete moment $t_{k}^{i}$ as the initial state, set up the length of planned horizon and seek the optimal control sequence $u_{k}$ of system in such planned horizon. Through acting the first item of control sequence $u_{k}$ to the system, we get new state of system and repeat executing the above operation by taking it as the initial condition, until the system state reaches the requirement of end constraint ${ }^{[6][7]}$. Then, we map the schematic diagram to express the theory vividly. 


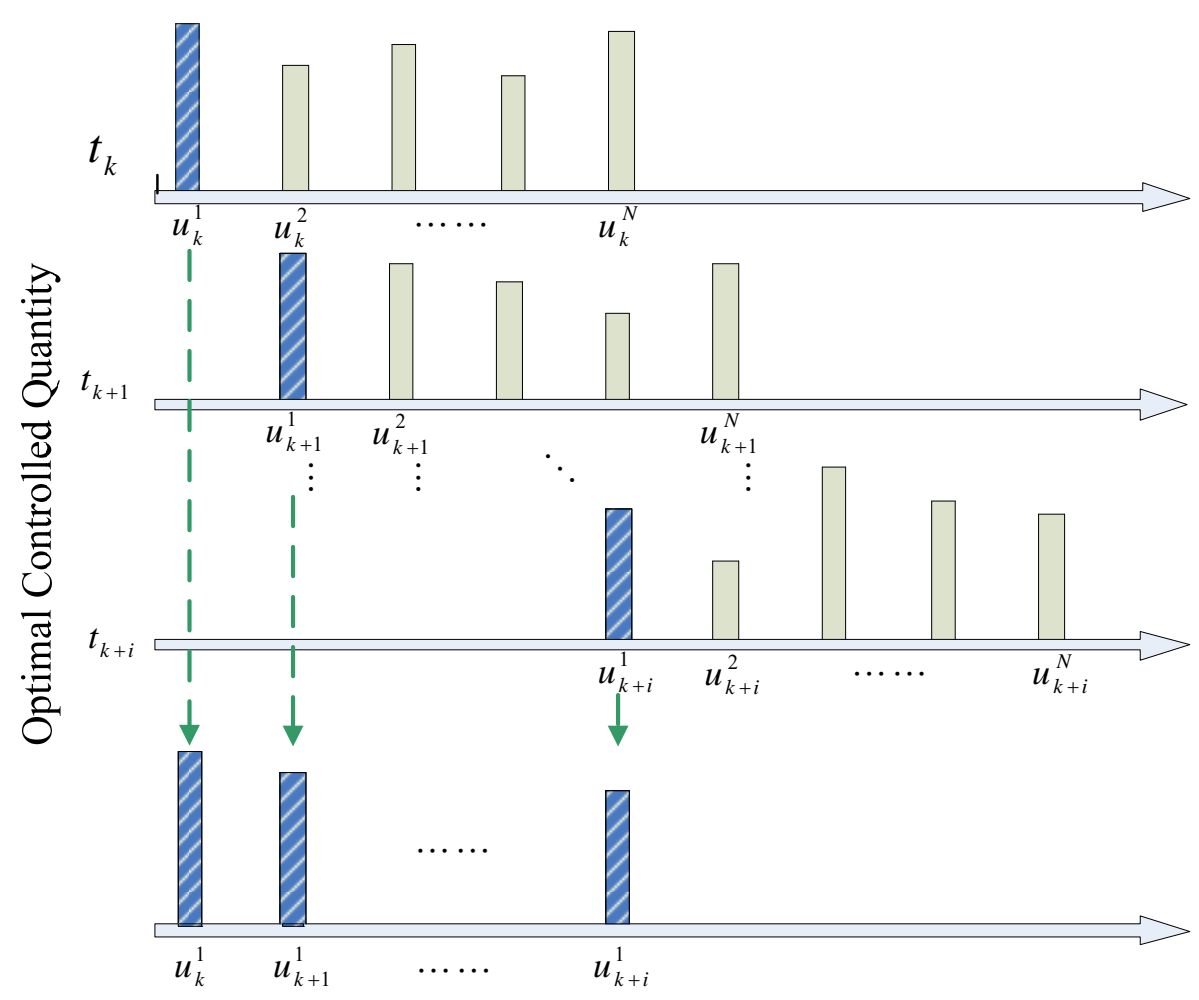

Figure 1 Basic thought of RHC.

PSO is a bionic intelligence algorithm proposed in the middle of $1990 \mathrm{~s}^{[8]}$. In n-dimension searching space, each particle is deemed as a point in the space. Meanwhile, each particle can be deemed as a potential solution of the problem to be solved. We suppose there are $\mathrm{m}$ particles in the group, $\mathrm{m}$ is called group size. In $t$ th iteration, we suppose the position of $i$ th particle is $X_{i}=\left(x_{i 1}, x_{i 2}, \cdots, x_{i n}\right)$.According to the specified fitness function previously, we compute the advantage and disadvantage of the current position; $V_{i}=\left(v_{i 1}, v_{i 2}, \cdots, v_{i n}\right)$ is the flight velocity of particle $i$, namely the mobile distance of particle; $P_{i}^{t}=\left(p_{i 1}, p_{i 2}, \cdots, p_{i n}\right)$ is the optimal position pbest passed by particle individual at present; $G_{i}^{t}=\left(g_{i 1}, g_{i 2}, \cdots, g_{i n}\right)$ expresses the optimal position of the searched group at the current position $g_{\text {best }}$ in the overall particle swarm.

In the iteration each time, the particle speed and position are updated as below:

$$
\begin{gathered}
v_{\text {id }}(t+1)=\omega v_{\text {id }}(t)+c_{1} r_{1}\left(p_{\text {id }}(t)-x_{\text {id }}(t)\right)+c_{2} r_{2}\left(g_{\text {id }}(t)-x_{\text {id }}(t)\right) \\
x_{\text {id }}(t+1)=x_{\text {id }}(t)+v_{\text {id }}(t)
\end{gathered}
$$

Hereinto, $\omega$ is inertia weight; $c_{1}$ and $c_{2}$ are learning factor, we enable the particle to approximate the optimal position $p_{\text {best }}$ passed by itself and the optimal position $g_{\text {best }}$ of group so as to summarize itself and learn from excellent individual in the group; $r_{1}$ and $r_{2}$ are a stochastic number between 0 and 1. These two parameters are to keep the diversity of group. In Equation (4.11), the second part expresses particle's learning of itself; the third part expresses the inter-particle coordination. Based on the above two kinds of learning thought, it makes the particle approach to a higher position of new fitness.

\subsection{Implementation Process of RHC-PSO Algorithm}

\subsubsection{Discretization Plan Horizon}

Hereinto, $q$ is growth rate of time step. Through the above discretion and combined with optimal control model proposed in 3.2, we can express the discretion of the optical control model in a planned horizon as: 


$$
\begin{array}{ll}
\min & J^{N}=\sum_{i=0}^{N-1} g\left(\boldsymbol{x}_{i}, \boldsymbol{u}_{i}, t_{k}^{i}\right)\left(t_{k}^{i+1}-t_{k}^{i}\right)+\rho\left(\boldsymbol{x}_{N}, t_{k}^{N}\right) \\
\text { s.t } & \boldsymbol{x}\left(t_{k}\right)=\boldsymbol{x}_{k} \\
& \boldsymbol{x}_{j+1}=A \boldsymbol{x}_{j}+B \boldsymbol{u}_{j}, \quad \forall j=0, \cdots, N-1 \\
& \mathrm{~S}\left(\boldsymbol{x}_{i}, \boldsymbol{u}_{i}, t_{k}^{i}\right) \leq 0
\end{array}
$$

To simplify the calculation, we select $N=2$ in the simulation calculation. Combined with the thought of RHC, we solve the optimal controlled quantity of two stage in the planned horizon and only select that acquired at the first stage to act on the system.

\subsubsection{Coding System of Controlled Quantity and Basic Process of RHC-PSO Algorithm}

The optimal controlled quantity in each planned horizon is solved using PSO. Decimal coding system is adopted in this paper, that is to say, the information of each particles is expressed with 6 decimal numbers (0-9), the first three numbers express the controlled quantity at the first stage and the latter three numbers express the controlled quantity at the second stage. We code the controlled quantity of the two stages in the planned horizon, and the coding system is as below ${ }^{[4]}$ :

Table 1 Coding system of controlled quantity.

\begin{tabular}{|c|c|c|c|c|c|c|}
\hline $\begin{array}{c}\text { Controlled } \\
\text { quantity }\end{array}$ & $\alpha_{1}$ & $\mu_{1}$ & $\eta_{1}$ & $\alpha_{2}$ & $\mu_{2}$ & $\eta_{2}$ \\
\hline Coding & $N_{1}$ & $N_{2}$ & $N_{3}$ & $N_{4}$ & $N_{5}$ & $N_{6}$ \\
\hline
\end{tabular}

Through a certain method, we transform the coding to controlled quantity (decoding). Taking controlled quantity $\alpha$ for an example, the transformation formula is as below:

$$
\alpha_{k}(t)=\alpha_{k-1}+\frac{\left(N_{3(k-1)+1}-4.5\right)}{4.5}\left(t-t_{0}\right) \Delta \alpha, \quad k=1,2
$$

$\Delta \alpha$ expresses the change rate of AOA. When the solved AOA is greater than the maximum AOA of aerial carrier, the controlled quantity of AOA selects maximum; when it is smaller than minimum value, we select minimum. Other controlled quantity is also decoded according to the method.

Basic procedure of PSO is as below:

Step 1: Set up maximum times of iteration, group size, inertia weight and learning factor.

Step 2: Initialize the position $X_{i}$ and velocity $V_{i}$ of each particle and

Step 3: Compute the fitness of each particle according to Formula (14), and initialize $p_{\text {best }}$ and $\mathrm{g}_{\text {best. }}$.

Step 4: Compare fitness of each particle with current $p_{\text {best }}$ and assign the better value to $p_{\text {best. }}$

Step 5: Compare fitness of each particle with current $g_{\text {best }}$ and assign the better value to $g_{\text {best. }}$

Step 6: Update the position and velocity of particle according to Equation (12) and (13).

Step 7: Judge whether the fitness of each particle meets the requirement or reaches the maximal items of iteration. If yes, move on to next step; if no, go back to Step 3.

Step 8: Take the current $g_{\text {best }}$ as the optimal solution.

\section{Simulation}

Performance of fighter: the minimal RCS of fighter is $0.15 \mathrm{~m}^{2}$ and the maximal RCS is $1.25 \mathrm{~m}^{2}$; the mass of fighter is $\mathrm{m}=2000 \mathrm{~kg}, \mathrm{~S}=50 \mathrm{~m}^{2}$.restrained by fighting task and self-performance of fighter, various controlled quantities and quantity of state of fighter in executing task are required as below: $100 \leq v \leq 350 ; 2^{\circ} \leq \gamma \leq 90^{\circ} ;-180^{\circ} \leq \gamma \leq 180^{\circ} ;-2^{\circ} \leq \alpha \leq 15^{\circ} ;-60^{\circ} \leq \mu \leq 60^{\circ} ; 0 \leq \eta \leq 1$. The initial state of fighter is as below: $t_{0}=0 ; v_{0}=256 ; \gamma_{0}=0^{\circ} ; \alpha_{0}=6^{\circ} ; \mu_{0}=0^{\circ} ; \eta_{0}=0.2$. 
End constraint: the fighter enters the above-mentioned weapon launching area.

Algorithm parameter: for weight of $W_{1}, W_{2}, W_{3}$ and $W_{4}$, we select $(0.4,0.2,0.2$ and 0.2$) ; N=2$; growth rate of step size $q=1 \mathrm{~s}$; length of planned horizon $T=3 \mathrm{~s}$; simulation step size is $0.1 \mathrm{~s}$; the decision execution time $t_{z}=0.5 \mathrm{~s}$; number of particle $m=50$, inertia weight $\omega=0.5$, learning factor $c_{1}=c_{2}=1.5$, and the maximal number of iteration is 50 . Through simulation in MATLAB environment, we get the following result:

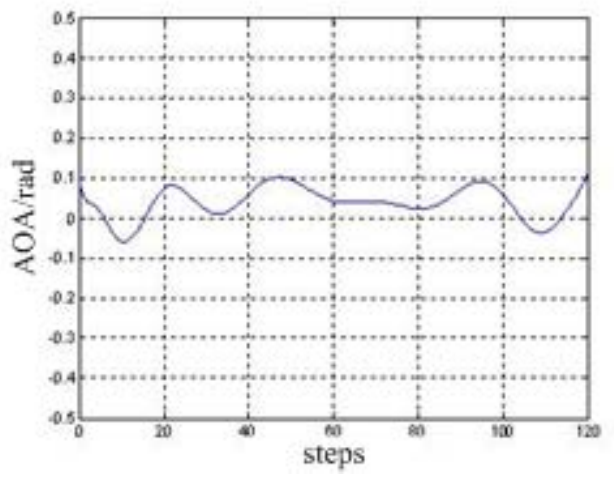

Figure 2 AOA change law

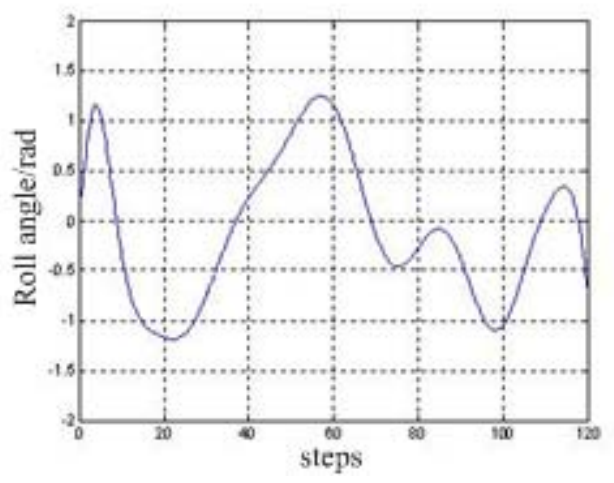

Figure 3 Roll angle change law

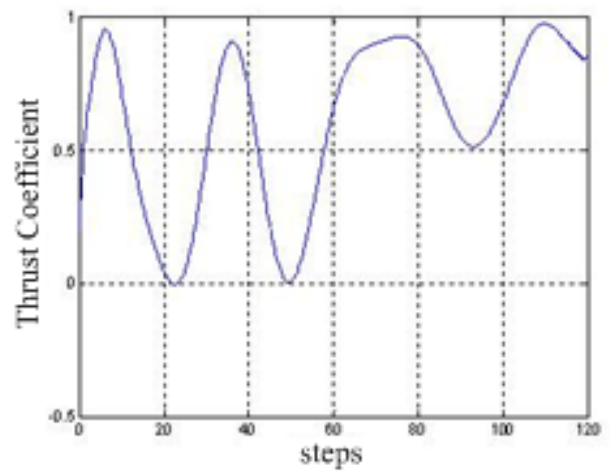

Figure 4 Thrust coefficient angle change law

Figure 2-4 shows the change law of AOA, roll angle and thrust coefficient with the change of decision times. It brings manipulation reference to pilot so as to provide assistance for pilot in trajectory control. 


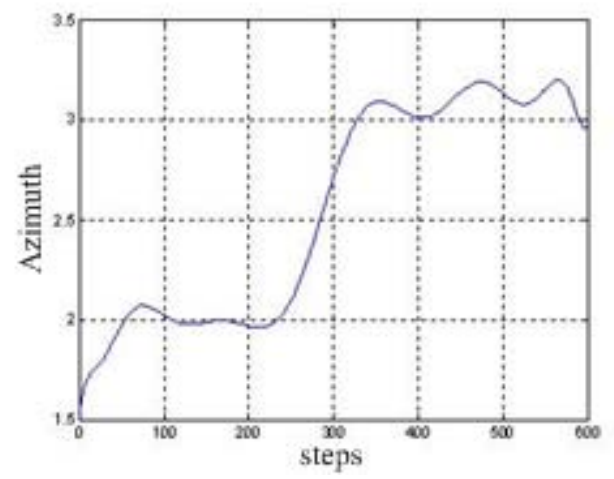

Figure 5 Trajectory azimuth change law

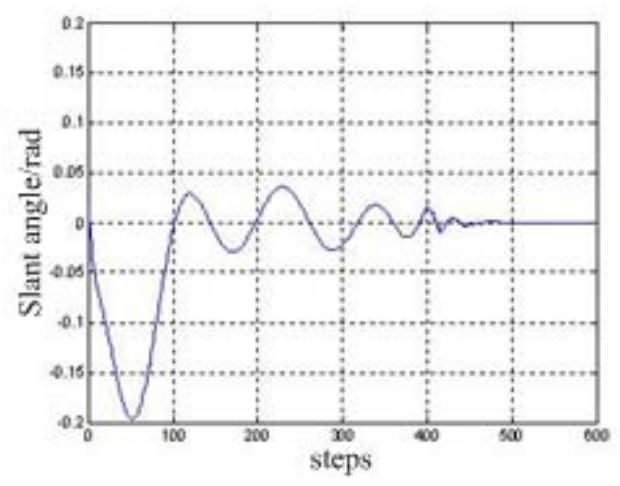

Figure 6 Slant angle change law

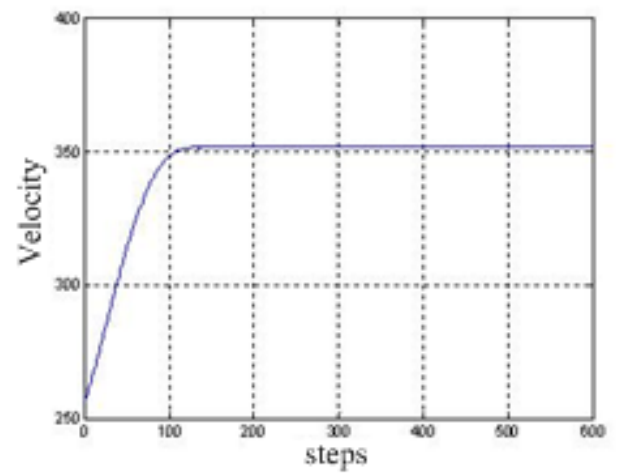

Figure 7 Velocity change law

Figure 5-7 expresses the change law of trajectory azimuth, slant angle of trajectory and velocity of aerial carrier. It is seen that the change of state quantity is gentle and the variation range is within the allowable range, which reflects the manipulability of the planned trajectory.

\section{Conclusion}

Against the trajectory control problem of air-ground attack, we select controlled quantity and quantity, determine the indicator function of trajectory control according to the requirement of fighting task and simplify various types of threat into constraint condition so as to transform trajectory control problem to optimal control problem. Then, we solved optimal value of controlled quantity in each decision horizon by using PSO and update horizon by using online receding of RHC so as to realize online real-time control. Through simulation, we verify that the method is practical and feasible, which provides subsidiary decision for pilot to manipulate aerial carrier and enhance the operational effectiveness. 


\section{References}

[1] ZHANG Yu, ZHANG Wanpeng. Air-to-ground Weapon Delivery Trajectory Planning for UCAVs Using Gauss Pseudospectral Method [J].Acts Aeronautica et Astronautics Sinica 2011.7,32(7):240-1251

[2] Huang Changqiang, Huang Hanqiao. Autonomous Combat Trajectory Optimization for UCAV (Unmanned Combat Aerial Vehicle) under Complex Uncertain Environment [J].Journal of Northwestern Polytechnical University, 2013.6,31(3):331-338

[3] Kai V, Janne K, Tuomas R. Modeling Air Combat by a Moving Horizon Influence Diagram Game[J]. Journal of Guidance, Control, and Dynamics, 2006, 29(5):1080-1091.

[4] ZHANG Tao, YU Lei, ZHOU Zhong-liang, WANG Lin, Decision-making for combat maneuvering based on hybrid algorithm [J]. Systems Engineering and Electronics, 2013, 35(7): 1445-1450.

[5] CHEN Lei. Intelligent Decision-making and Autonomous Control for Air-to-ground Attacking under Complex Uncertain Environment [D].Xi'an: Air Force Engineering University, 2011

[6] Zhan Z H, Zhang J, Li Y. An efficient ant colony system based on receding horizon control for the aircraft arrival sequencing and scheduling problem [J]. IEEE Transactions on Intelligent Transportation Systems, 2010, 11(2): 399-412.

[7] Anisi D A. On-line Trajectory Planning for Aerial Vehicles: a Safe Approach with Guaranteed Task Completion[R]. AIAA 2006-6107, 2006.

[8] JI Zhen, LIAO Huilian, WU Qinghua. Particle Swarm Optimization and Application [M]. Beijing: Science Press, 2009. 